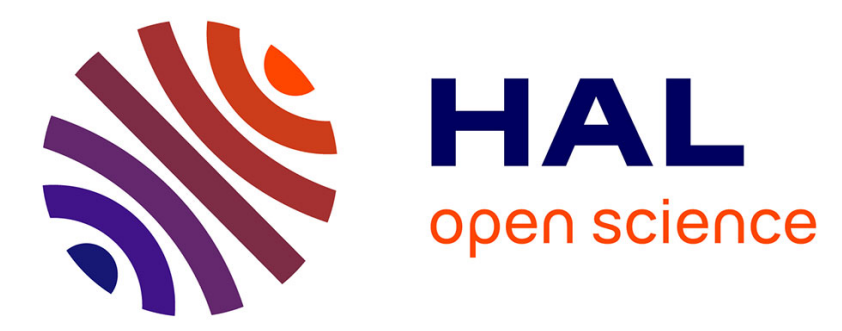

\title{
4K real time video streaming with SHVC decoder and GPAC player
}

Wassim Hamidouche, Gildas Cocherel, J. Le Feuvre, Mickaël Raulet, Olivier Deforges

\section{- To cite this version:}

Wassim Hamidouche, Gildas Cocherel, J. Le Feuvre, Mickaël Raulet, Olivier Deforges. 4K real time video streaming with SHVC decoder and GPAC player. Multimedia and Expo Workshops (ICMEW), 2014 IEEE International Conference on, Jun 2014, Chengdu, China. pp.1-2, 10.1109/ICMEW.2014.6890613 . hal-01068628

\section{HAL Id: hal-01068628 \\ https://hal.science/hal-01068628}

Submitted on 26 Sep 2014

HAL is a multi-disciplinary open access archive for the deposit and dissemination of scientific research documents, whether they are published or not. The documents may come from teaching and research institutions in France or abroad, or from public or private research centers.
L'archive ouverte pluridisciplinaire $\mathbf{H A L}$, est destinée au dépôt et à la diffusion de documents scientifiques de niveau recherche, publiés ou non, émanant des établissements d'enseignement et de recherche français ou étrangers, des laboratoires publics ou privés. 


\title{
4K REAL TIME VIDEO STREAMING WITH SHVC DECODER AND GPAC PLAYER
}

\author{
W. Hamidouche, G. Cocherel, J. Le Feuvre, M. Raulet and O. Déforges \\ IETR/INSA, Rennes, France and Télécom Paris Tech, Paris, France \\ Wassim.Hamidouche@insa-rennes.fr
}

\begin{abstract}
This paper presents the first $4 \mathrm{Kp} 30$ end-to-end video streaming demonstration based on the upcoming Scalable High efficiency Video Coding (SHVC) standard. The optimized and parallel SHVC decoder is used under the GPAC player to decode and display in real time the received SHVC layers. The SHVC reference software model (SHM) is used to encode the $4 \mathrm{~K}$ original video in two spatial scalability layers: the base layer at 1080p resolution and the enhancement layer at 2160p resolution. The SHVC bitstream is encapsulated with the GPAC multimedia library into MP4 file format. The GPAC player at the server side broadcasts the MP4 content in MPEG-2 TS. At the client side, the GPAC player receives the SHVC video packets which are decoded by the SHVC decoder and then rendered in real time by the player. The GPAC player provides an interactive interface enabling to switch between displaying the base and the enhancement layers.
\end{abstract}

Index Terms - End-to-end 4K video streaming, SHVC standard, parallel processingn, DVB-T2, DVB-S2.

\section{INTRODUCTION}

The Joint Collaborative Team on Video Coding (JCT-VC) defines the SHVC standard to enabling temporal, spatial and quality (SNR) scalability [1]. The SHVC extension is expected to be finalized during the next JCT-VC meeting in July 2014. The SHVC standard is based on the HEVC technologies and uses inter-layer predictions to take advantage of the spatial correlations. The SHVC coding into two layers introduces a rate-distortion loss of only $10 \%-30 \%$ in respect to the simulcast HEVC coding configuration [2]. Moreover, the SHVC standard supports the base layer coded in the legacy AVC standard. Therefore, SHVC extension can be an attractive solution for several video streaming applications, including UHD-TV broadcasting and IPTV or HTTP streaming for UHD-TV. Figure 1 shows the SHVC video transmission over satellite and terrestrial broadcast networks. The idea behind this use case is to transmit at high bitrate two SHVC layers in separated PIDs over the satellite channel for full HD and $4 \mathrm{~K}$ resolutions, respectively. Thus, end-users with satellite

This work is funded by the national FUI project OPTISAT2.

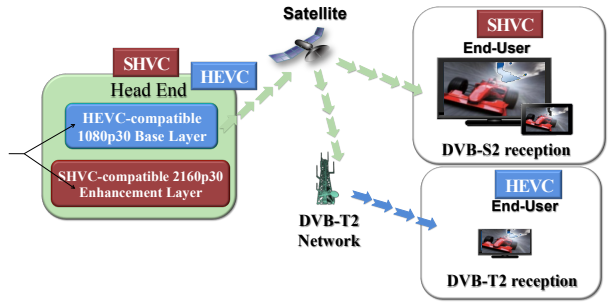

Fig. 1. SHVC broadcast over satelite and teresterial channels

receiver can decode both layers enabling to display the programme in $4 \mathrm{~K}$ resolution. On the other hand, only one PID containing the base layer is retransmitted at lower biterate through the terrestrial channel. This enables end-uses connected to only terrestrial network to display the programme in full HD resolution. This use-case uses SHVC capability to adapt the bitrate of the video content to the channel bandwidth constraint, and avoids using resource-consuming transcoding solutions. This paper describes the demonstration of this use case on a computer with the SHVC x 2 spatial scalability configuration: 1080p30 base layer and 2160p30 enhancement layer. This demonstration is carried out using only our open source software projects including the GPAC multimedia library [3] and the optimized SHVC decoder [4]. First, the original $4 \mathrm{~K} 2 \mathrm{~K}$ video is encoded with the SHVC reference software model version 4.1 (SHM) [5] into two $\mathrm{x} 2$ spatial scalability layers (full HD and 4K). The elementary SHVC stream is then encapsulated in two tracks MP4 file format with the GPAC player. At the server side, the GPAC player broadcasts the mp4 file format including the two tracks over the network in MPEG-2- TS format. At the client side, the GPAC player feeds the SHVC decoder with the received layers. The SHVC decoder decodes the two layers and pushes the decoded YUV picture corresponding to the highest layer to the GPAC player for rendering. The GPAC player can emulate the end-users connected to terrestrial or satellite networks by decoding only the base layer or the two layers, respectively.

\section{OPTIMIZED SHVC DECODER}

The received layers are decoded in real time with the optimized SHVC decoder. We developed the SHVC decoder 


\begin{tabular}{lcc|cc}
\hline \multirow{2}{*}{ Sequences } & \multicolumn{2}{c|}{ Bitrate (Mbps) } & \multicolumn{2}{c}{ Decoding frame rate (fps) } \\
\cline { 2 - 5 } & BL & EL & BL (HD) & BL+EL (4K) \\
\hline \multirow{2}{*}{ CrowdRun } & 8.23 & 15.15 & 138 & 43 \\
& 14.66 & 27.36 & 110 & 35 \\
\hline \multirow{2}{*}{ ParkJoy } & 9.94 & 21.91 & 134 & 41 \\
& 19.68 & 41.69 & 93 & 33 \\
\hline Average & 13.12 & 26.52 & 118 & 38 \\
\hline
\end{tabular}

Table 1. Performance of the optimized and parallel SHVC decoder

on the top of the OpenHEVC project [4] which implements a conforming HEVC decoder. The SHVC decoder consists of independent OpenHEVC decoders, one decoder to decode each layer. A communication mechanism is implemented to perform the inter-layer predictions between the HEVC decoders. All decoding operations including motion compensation, inverse transform and up-sampling filters are optimized in Single Instruction Multiple Data (SIMD) Intel SSE methods for x86 architecture. Moreover, the SHVC decoder is friendly-parallel and leverages multi-core architecture to improve both the decoding frame rate and the decoding frame latency. The SHVC decoder supports a hybrid parallelism solution combining the wavefront parallel processing solution with the frame-based parallelism. This hybrid parallelism solution performs a high speedup performance with a good trade-off between decoding frame rate, decoding latency and memory usage [6]. Table 1 shows the performance of the SHVC decoder on a laptop fitted with a 4 Cores Intel i7 processor running at $2.3 \mathrm{GHz}$ for two $4 \mathrm{~K}$ video sequences.

\section{4K END-END SHVC VIDEO STREAMING}

In this section we provide the operation required to run the demonstration using the GPAC player and the SHVC decoder on Windows OS. The GPAC installer release 5200 can be downloaded from [3]. GPAC provides all executables used in this demonstration including MP4Box, MP4Client and $m p 42 t s$. The $4 \mathrm{~K}$ SHVC video bitsreams coded in two $\mathrm{x} 2$ spatial scalability configuration are attached to this paper. These bitsreams can also be encoded with using the reference software SHM4.1 [5] with the following command line:

TAppEncoderStatic -c/cfg/encoder_lowdelay_main.cfg -c /cfg/per-sequence-svc/ParkJoy-2x.cfg -q0 30 -q1 30 - NumLayers $=2-$ WaveFrontSynchro $=1$

The SHVC bitstream can be encapsulated into two tracks MP4 file format using the MP4Box executable as follows:

MP4Box -new -add ParkJoy_2160p.shvc:fps=30:svcm ode $=$ split ParkJoy2160.mp4

In this example the server and the client run at the same computer in localhost mode. This command line enables the server to stream the two SHVC layers:

mp42ts - prog= ParkJoy2160.mp4 -single-au -dst-udp= 224.1.1.1:1234

The client can decode and display the received SHVC layers with the following command line:

$$
\text { MP4Client mpegts-udp://224.1.1.1:1234 }
$$

The default configuration displays the highest decoded layer. However, the client can switch between the base and the enhancement layers by $c t r+l$ and $c t r+h$, respectively. Figure 2 illustrates the proposed demonstration displaying both $\mathrm{HD}$ (on the left) and $4 \mathrm{~K}$ video sequence on a 84 inches $4 \mathrm{~K}$ TV.

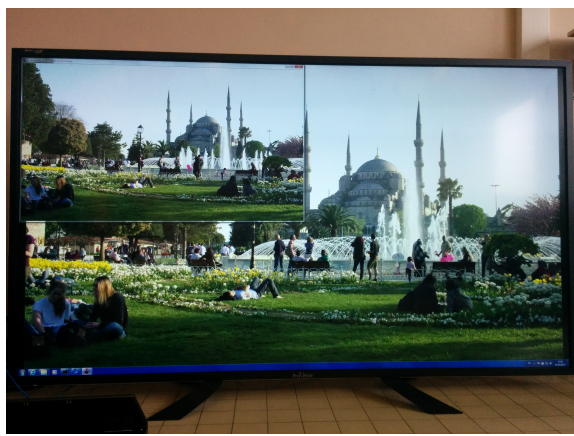

Fig. 2. GPAC player displaying $4 \mathrm{~K}$ and $\mathrm{HD}$ video sequences on a 84 inches $4 \mathrm{~K} \mathrm{TV}$

\section{REFERENCES}

[1] J. Chen, J. Boyce, Y. Ye, and M. M. Hannuksela, "Scalable HEVC (SHVC) Test Model 4 (SHM 4)," in document JCTVC-O1007. Geneva, Switzerland, November 2013.

[2] V. Seregin, T. D. Chuang, Y. He, D. K. Kwon, and F. Le Leannec, "AHG Report: SHVC software," in document JCTVC-L0011. Geneva, Switzerland, January 2013.

[3] "GPAC multimedia open source project," in http://gpac.wp.mines-telecom.fr.

[4] "Open source HEVC decoder (OpenHEVC)," in https:://github.com/OpenHEVC.

[5] "SHVC Reference software model (SHM)," in https://hevc.hhi.fraunhofer.de/svn/svn_SHVCSoftware/.

[6] W. Hamidouche, M. Raulet, and O. Deforges, "Parallel SHVC Decoder: Implementation and Analysis," in IEEE conference on ICME. Chengdu, China, July 2014. 